\title{
A Review of Rhazya stricta Decne Phytochemistry, Bioactivities, Pharmacological Activities, Toxicity, and Folkloric Medicinal Uses
}

\author{
Abdulaziz Albeshri ${ }^{1, *(\mathbb{D})}$, Nabih A. Baeshen ${ }^{1}$, Thamer A. Bouback ${ }^{1,2}$ and Abdullah A. Aljaddawi ${ }^{1}$ \\ 1 Department of Biological Science, Faculty of Science, King Abdul-Aziz University, \\ Jeddah 21589, Saudi Arabia; nabih_baeshen@hotmail.com (N.A.B.); tbouback@kau.edu.sa (T.A.B.); \\ aaljaddawi@kau.edu.sa (A.A.A.) \\ 2 Princess Dr. Najla Bint Saud Al-Saud Center for Excellence Research in Biotechnology, \\ King Abdul-Aziz University, Jeddah 21589, Saudi Arabia \\ * Correspondence: aalbishri0061@stu.kau.edu.sa
}

Citation: Albeshri, A.; Baeshen, N.A.; Bouback, T.A.; Aljaddawi, A.A. A Review of Rhazya stricta Decne Phytochemistry, Bioactivities, Pharmacological Activities, Toxicity, and Folkloric Medicinal Uses. Plants 2021, 10, 2508. https://doi.org/ $10.3390 /$ plants10112508

Academic Editor: Ki Hyun Kim

Received: 19 October 2021

Accepted: 8 November 2021

Published: 19 November 2021

Publisher's Note: MDPI stays neutral with regard to jurisdictional claims in published maps and institutional affiliations.

Copyright: (c) 2021 by the authors. Licensee MDPI, Basel, Switzerland. This article is an open access article distributed under the terms and conditions of the Creative Commons Attribution (CC BY) license (https:// creativecommons.org/licenses/by/ $4.0 /)$.

\begin{abstract}
The local medicinal plant Rhazya stricta Decne is reviewed for its folkloric medicinal, phytochemical, pharmacological, biological, and toxicological features. $R$. stricta has been used widely in different cultures for various medical disorders. The phytochemical studies performed on the R. stricta extract revealed many alkaloidal and fatty acid compounds. Moreover, several flavonoid and terpenoid compounds were also detected. Pharmacological activates of $R$. stricta extracts are approved to possess antimicrobial, antioxidant, anticancer, antidiabetic, and antihypertensive activities. Additionally, R. stricta extract was found to hold biological activates such as larvicidal and phytoremediation activates $R$. stricta extract was found to be toxic, genotoxic, and mutagenic. R. stricta contains novel phytochemical compounds that have not been investigated pharmacologically. Further research is needed through in vitro and in vivo experiments to pave the road for these compounds for medical, veterinary, and ecological uses.
\end{abstract}

Keywords: Rhazya stricta; folkloric medicine; alkaloid; phytochemicals; pharmacological activity; toxicity

\section{Introduction}

R. stricta is one of the most economically valuable medicinal plants found throughout arid South Asia and the Arabian Peninsula. Leaf extracts were traditionally utilized in the treatment of a wide variety of illnesses, such as syphilis, parasitic infections, hyperglycemia, and rheumatism, as well as the common cold [1]. The Rhazya stricta species was labeled after one Muslim scientist known as Mohammed bin Zakariya AlRazi (925), and it is generally recognized in Europe as Rhazes [2]. Numerous studies used various parts of $R$. stricta extract to screen for phytochemical constituents. Over a hundred alkaloids and many compounds belonging to other groups, such as flavonoids and lipids, have been isolated [3,4]. It has been proven that the alkaloidal compounds possess multiple activities, including antitumor, antimicrobial, and antihypertensive [5]. The main objective of this review is to provide advanced and updated information about R.stricta plant research.

\section{Methodology}

The content for this review was extracted from Google Scholar articles. The scientific name "Rhazya stricta" was used to cover all relevant data from 1800-2021, including traditional uses, nutritional compositions, phytochemical compounds, and pharmacological properties (anticancer, antioxidant, antiviral, antimicrobial, anti-inflammatory, hepatoprotective, antidiabetic, and antihypertensive) of the plant described in this review. 


\section{Regional Names}

In Urdu, it is referred to as "Rangobul," "Vergalum," "Ganderi" in Pushto, and "harmal" in Arabic. It is important, however, to distinguish the harmal for Peganum harmala from the harmal for $R$. stricta [2].

\section{Regional Distribution}

R. stricta is distributed throughout Southwest Asia (India, Pakistan, and Afghanistan) and the countries bordering the Arabic gulf, e.g., Saudi Arabia, the United Arab Emirates (UAE), Iraq, Iran, and Qatar [6] (Figure 1).

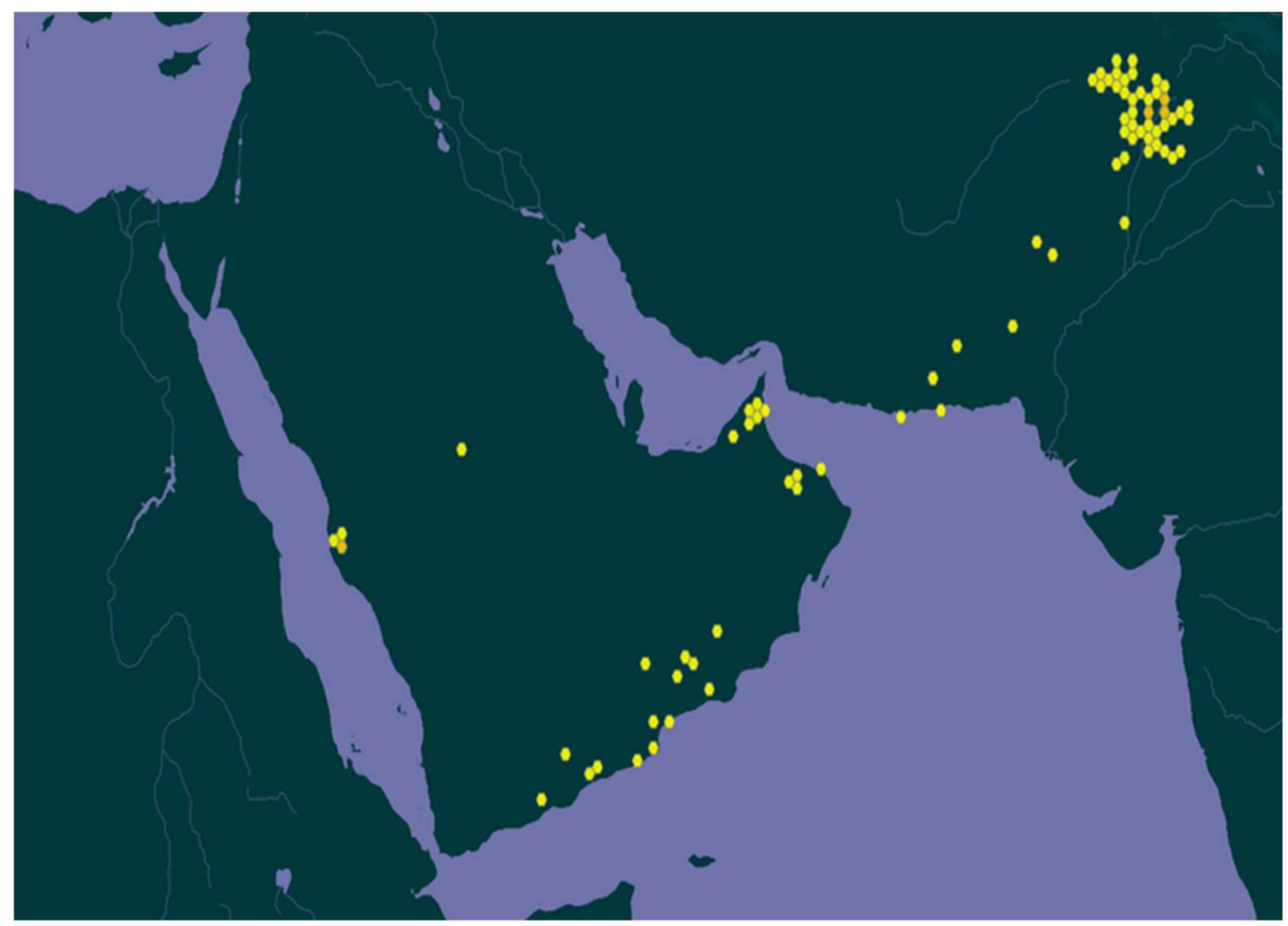

Figure 1. Map shows the regional distribution of the R. stricta plant [7].

\section{R. stricta Taxonomy}

R. stricta is a species belonging to the Apocynaceae family. The Apocynaceae family contains 424 genera and over 4600 species, which are classified into five subfamilies: Apocynoideae, Periplocoideae, Rauvolfioideae, Asclepiadoideae, and Secamonoideae [8] (Table 1).

Table 1. R. stricta taxonomy according to Global Biodiversity Information Facility [7].

\begin{tabular}{cc}
\hline Kingdom & Plantae \\
\hline Phylum & Tracheophyta \\
\hline Class & Magnoliopsida \\
\hline Order & Gentianales \\
\hline Family & Apocynaceae \\
\hline genes & Rhazya Decne \\
\hline species & Rhazya stricta Decne \\
\hline
\end{tabular}




\section{Morphology Characteristics}

$R$. stricta is an evergreen miniature shrub with thick foliage. It is a glabrous, upright perennial plant with many branches emerging from the base. The main stalk is smooth and thickly branched, particularly towards the base, in a semi-erect form. Sessile and simple leaf are linear-oblong or ellipsoidal, practically upright, with an entire border and sharp apex, dense, leather, and parallel blade tapering toward the base. Flowers are bisexual; inflorescences are axillary cymes found near the tips of branches; flowers are pentamerous, white, 2-2.5 cm long, short-pedicelled, and have inserted stamens; Flowers are heterosexual; inflorescences are axillary cymes located towards the ends of branches; flowers are pentamerous, white, $2-2.5 \mathrm{~cm}$ in length, short-pedicelled, and have attached stamens; flowers have white petals; the calyx is roughly $4 \mathrm{~mm}$ long, deeply lobed. The corolla is $1-1.4 \mathrm{~cm}$ in diameter, white; tube cylindrical; lobes ovate, with a rounded mucronate apex, c. 12-15 mm long, and have a brownish-green tube enlarged slightly above the middle and longer than the salverform limb, partially occluded by bristles at the throat; limb lobes are widely obovate, obtuse, mucronate, and are white inside [4] (Figure 2).

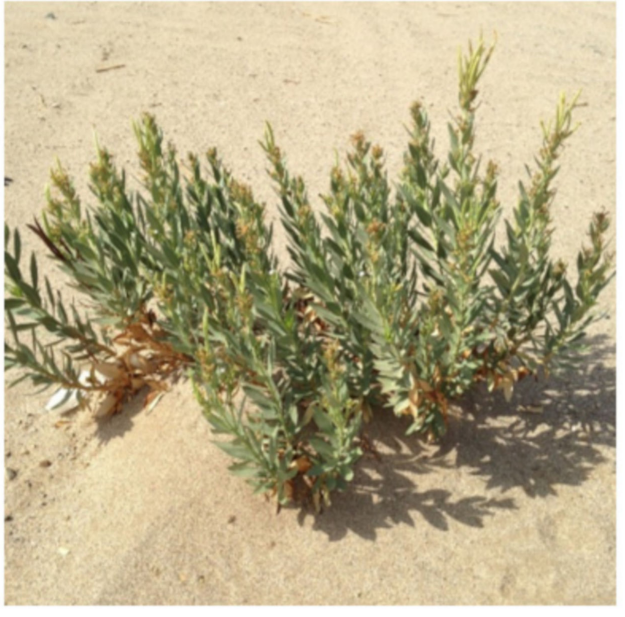

(A)

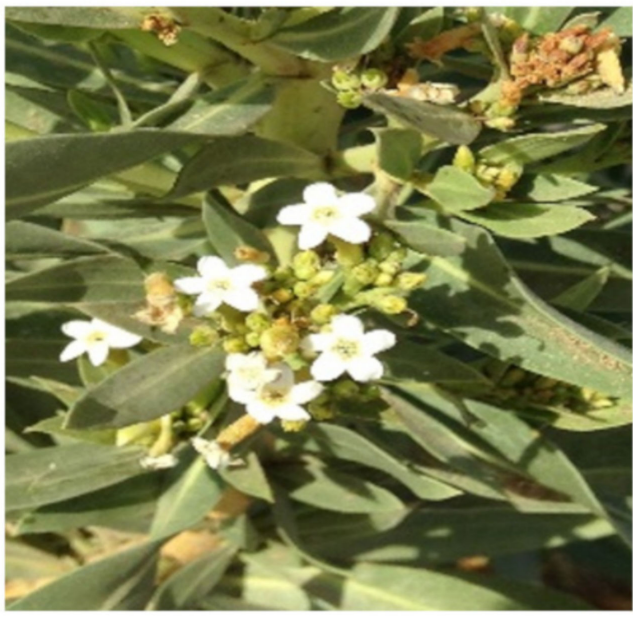

(C)

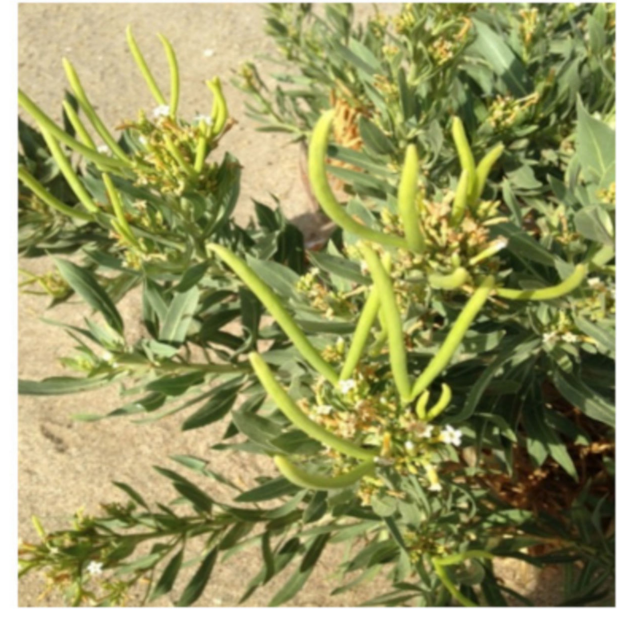

(B)

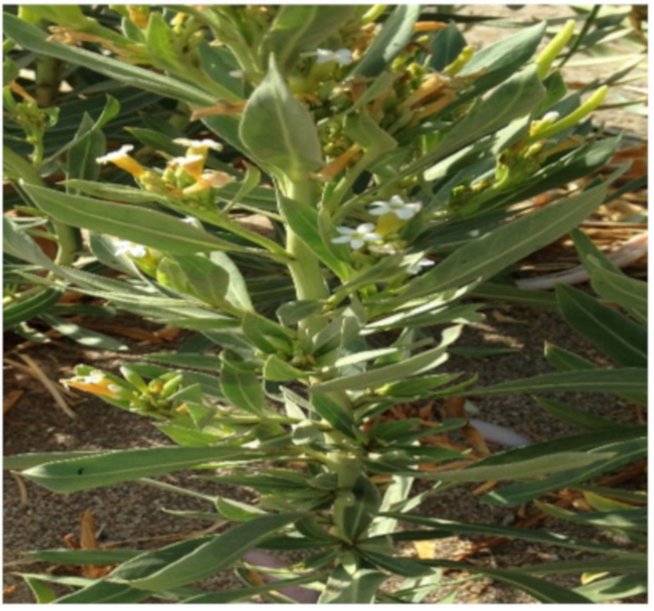

(D)

Figure 2. Photos captured of (A) whole plant of R. stricta or its parts (B) fruits, (C) flowers, and (D) stem and leaves from Wadi Fatimah, Makkah.

\section{Folkloric Medicinal Uses of R. stricta}

$R$. stricta leaves are exploited in the traditional system of medicine in rural areas of Saudi Arabia to treat syphilis, chronic rheumatism, and body pain. [8]. Local folk medicine 
practitioners utilize $R$. stricta to treat type 2 diabetes, certain inflammatory disorders, helminthiasis, and sore throat $[9,10]$. In Pakistan, $R$. stricta extract used to treat pimples and acne on the face. Additionally, fresh leaves are preserved in footwear and placed under the soles to alleviate foot burn and treat rheumatic disorders. Healers in Oman treat chest pain, conjunctivitis, constipation, and a variety of other ailments with $R$. stricta $[11,12]$.

\section{Content Properties of R. stricta Extract}

Several studies have exerted their efforts on discovering the alkaloid compounds, and they also discovered some non-alkaloid compounds [13-24] (Figure 3). A study estimated the contents of $R$. stricta extracts; the root contains the most alkaloids $(3.5 \mathrm{~g} / 100 \mathrm{~g})$, while the leaves contain the most tannins, phenolic compounds, and antioxidants, $(0.64 \mathrm{~g} / 100 \mathrm{~g})$, $(1.4 \mathrm{~g} / 100 \mathrm{~g})$, and $(0.56 \mathrm{~g} / 100 \mathrm{~g})$, respectively. The stems contain a high concentration of flavonoids (0.74 g/100 g) [25]. Another study examined the metal content of $R$. stricta extract and discovered the presence of several metals, including $\mathrm{Fe}, \mathrm{Cd}, \mathrm{Ar}, \mathrm{Ma}, \mathrm{Ca}, \mathrm{Cr}, \mathrm{Cd}$, $\mathrm{Ni}, \mathrm{Pb}, \mathrm{K}, \mathrm{Na}$, and $\mathrm{Cu}[26]$. A study determined that $R$. stricta is rich in a variety of alkaloids, flavonoids, polyphenols, tannins, and many other phytochemicals by analyzing the functional groups identified in the root extract [27]. Saponins, tannins, alkaloids, flavonoids, and polyphenols were detected in aqueous extract $R$. stricta during the phytochemical analysis [28] (Tables 2 and 3).

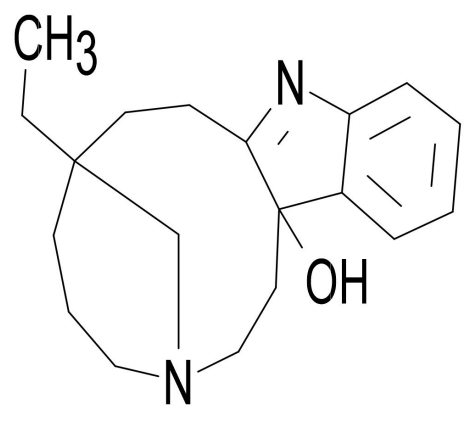

(a)<smiles>C/C=C1/CN2c3c([nH]c4ccccc34)CC3C2CC1CC3C(CO)(CO)C(=O)OC</smiles>

(d)<smiles>C/C=C1\CN2CCc3c([nH]c4ccccc34)C2CC1C(CO)C(=O)OC</smiles>

(b)

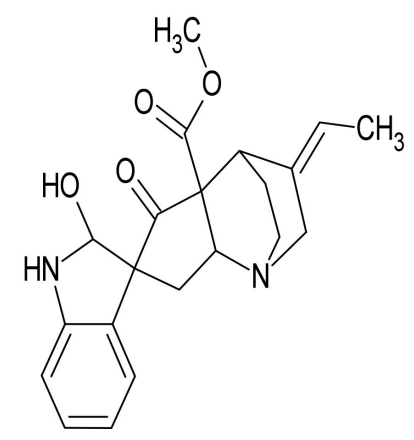

(e)<smiles>C/C=C1\CN2CCC34C=Nc5ccccc5C3(C=N4)C2CC1C(=O)OC</smiles>

(c)

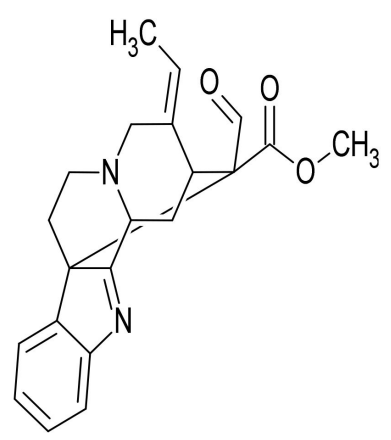

(f)

Figure 3. Chemical structures of some alkaloidal compounds (a) Rhazidigenine, (b) Rhazimanine, (c) Rhazimine, (d) Rhazine, (e) Rhazicine, and (f) Rhazimal named after the R. stricta plant [29]. 
Table 2. List of R. stricta compounds identified and extracted using the PubChem data base [29].

\begin{tabular}{|c|c|c|c|}
\hline Ref & CID & Compounds & \\
\hline [2] & 15558574 & Akuammidine (Rhazine) & 1. \\
\hline [2] & 5462421 & Antirhine & 2. \\
\hline [2] & 5491661 & 3-epi-Antirhine & 3. \\
\hline [2] & 580281 & Aspidospermidine & 4. \\
\hline [2] & 5378963 & Condylocarpine & 5. \\
\hline [2] & 164952 & Dihydrocorynantheol & 6. \\
\hline [2] & 6857502 & Eburnamenine & 7. \\
\hline [2] & 101699 & Eburnamine & 8. \\
\hline [2] & 92112 & Eburnamonine & 9. \\
\hline [2] & 5280491 & Geissoschizine & 10. \\
\hline [2] & 6436828 & Isositsirikine & 11. \\
\hline$[2,30]$ & 5377267 & 16-Epi-Z-isositsirikine & 12. \\
\hline [2] & 125060 & Leuconolam & 13. \\
\hline [2] & 160263 & Rhazinilam & 14 . \\
\hline [2] & 169527 & Tetrahydrosecamine & 15. \\
\hline [2] & 193109 & Presecamine & 16. \\
\hline [2] & 5458504 & Sewarine & 17. \\
\hline [2] & 10066724 & Stemmadenine & 18. \\
\hline [2] & 301805 & Strictamine & 19. \\
\hline [2] & 10345799 & Strictosamide & 20. \\
\hline [2] & 161336 & Strictosidine & 21. \\
\hline [2] & 20485 & Tabersonine & 22. \\
\hline [2] & 72340 & Tetrahydroalstonine & 23. \\
\hline [2] & 5384527 & Vallesiachotamine & 24 . \\
\hline [2] & 6443646 & Rhazimine & 25. \\
\hline [2] & 14109838 & Rhazimanine & 26. \\
\hline$[2,30]$ & 5318674 & Bhimberine & 27. \\
\hline [2] & 101607204 & Leepacine & 28. \\
\hline [2] & 12314912 & Rhazidigenine & 29. \\
\hline$[2,30]$ & 624708 & (+)-Quebranchamine & 30. \\
\hline$[2,30]$ & 579873 & (-)-Vincadiformine & 31. \\
\hline [2] & 102276348 & Secamine & 32. \\
\hline [2] & 12444819 & Vincadine & 33. \\
\hline [2] & 101407506 & Strictamine-N-oxide & 34. \\
\hline [2] & 624448 & 1,2-Didehydroaspidospermidine & 35. \\
\hline [2] & 102276826 & Dihydrosecamine & 36. \\
\hline [2] & 102276822 & Dihydropresecamine & 37. \\
\hline$[2,30]$ & 626805 & Rhazidigenine-N-oxide & 38. \\
\hline$[2,30]$ & 14825828 & Decarbomethoxy-15,20,16,17-tetrahydrosecodine & 39. \\
\hline [2] & 624449 & Dihydroeburnamenine & 40. \\
\hline
\end{tabular}


Table 2. Cont.

\begin{tabular}{|c|c|c|c|}
\hline Ref & CID & Compounds & \\
\hline$[2,30]$ & 5757451 & Nor-C-luorocurarine & 41. \\
\hline [2] & 5374154 & Polyneuridine & 42. \\
\hline$[2,30]$ & 540749 & (-)-16R,21R-Omethyleburmanine & 43. \\
\hline$[2,30]$ & 94255 & (-)-Vincadifformine & 44 . \\
\hline$[2,30]$ & $-\ldots-\ldots$ & Aspidospermiose & 45. \\
\hline$[2,30]$ & - - - - - - & Bhimberine-N-oxide & 46. \\
\hline$[2,30]$ & $-\ldots$ & Rhazicine & 47. \\
\hline$[2,30]$ & $-\ldots$ & 2-Methoxy 1-2, dihydrorhazamine & 48. \\
\hline$[2,30]$ & $-\ldots-\ldots-\ldots$ & 16-Hydrorhazisidine & 49. \\
\hline$[2,30]$ & - & Dihydrosecodine & 50. \\
\hline$[2,30]$ & 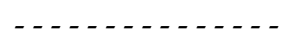 & HR-1 & 51. \\
\hline$[2,30]$ & - - - - - - - - - & N-methylleuconolam & 52. \\
\hline$[2,30]$ & $-\ldots \ldots$ & Rhazinaline & 53. \\
\hline$[2,30]$ & $-\ldots$ & Stricticine & 54 . \\
\hline$[2,30]$ & $-\ldots$ & Strictalamine & 55. \\
\hline$[2,30]$ & 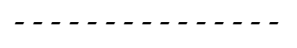 & Strictigine & 56. \\
\hline$[2,30]$ & - - - - - - - - - & Strictisidine & 57. \\
\hline$[2,30]$ & - - - - - - - - & Tetrahydrosecodine & 58. \\
\hline$[2,30]$ & $-\ldots$ & Vincanicine & 59. \\
\hline$[2,30]$ & $-\ldots-\ldots$ & Isorhazicine & 60. \\
\hline$[2,30]$ & $-\ldots$ & Rhazinol & 61. \\
\hline$[2,30]$ & - . - - - & Rhazimol & 62. \\
\hline$[2,30]$ & $-\ldots-\ldots-\ldots$ & Rhazizine & 63. \\
\hline$[2,30]$ & $-\ldots-\ldots$ & 15-Hydroxyvincadifformine & 64 . \\
\hline [2] & 177185 & $16 \mathrm{~s}, 16^{\prime}$-Decarboxytetrahydrosecamine & 65. \\
\hline$[2,30]$ & $-\ldots-\ldots-\ldots$ & Strictibine & 66. \\
\hline [2] & 101967159 & Rhazimal & 67. \\
\hline [2] & 12313538 & Vincanine & 68. \\
\hline [2] & 5280794 & Stigmasterol & 69. \\
\hline [2] & 222284 & $\beta$-Sitosterol & 70. \\
\hline [2] & 20756463 & Phytochelatins & 71. \\
\hline [2] & - - - - - - - - & Bis-strictidine & 72. \\
\hline [2] & - - - - - - - - & 1,2-Dehydroaspidospermine $\mathrm{N}$-oxid & 73. \\
\hline$[2]$ & - - - - - - - - - & 3, 14-Dehydrorhazigine & 74. \\
\hline [2] & 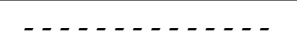 & Dihydroebumamenine & 75. \\
\hline$[2]$ & $\ldots \ldots \ldots$ & 21S-Ebumamenine & 76. \\
\hline$[2]$ & $-\ldots-\ldots$ & 16-Formylstrictamine & 77. \\
\hline [2] & - - - - - - - - & Harhingine & 78. \\
\hline$[2]$ & 11530478 & $15 \beta$-Hydroxyvincadifformine & 79. \\
\hline [2] & - - - - - - - - - & 16,hydroxyrhazisidine & 80. \\
\hline
\end{tabular}


Table 2. Cont.

\begin{tabular}{|c|c|c|c|}
\hline Ref & CID & Compounds & \\
\hline [2] & 6442678 & Isovallesiachotamine & 81. \\
\hline [2] & 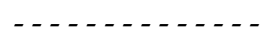 & 2-Methoxy 1,2- dihydorhazimine & 82. \\
\hline [2] & $-\ldots-\ldots$ & 17-Methoxy 1, 17-dihydorhazimine & 83. \\
\hline [2] & $-\ldots \ldots$ & 16R,21R-O-Methylebumamine & 84. \\
\hline$[2]$ & $-\ldots-\ldots-\ldots$ & N-Methyleuconulam & 85. \\
\hline [2] & 5581319 & Norfluorocurarine & 86. \\
\hline [2] & $-\ldots-\ldots$ & $\mathrm{N} \beta$-methyl strictamine & 87. \\
\hline [2] & $-\ldots-\ldots$ & Rhazigine & 88. \\
\hline [2] & $\ldots \ldots \ldots$ & Rhazimidine & 89. \\
\hline [2] & - . - . - - & Rhazinol & 90. \\
\hline [2] & - - - - - - - - & Rhazind & 91. \\
\hline$[2,30]$ & 21725847 & Rhazinine & 92. \\
\hline [2] & $-\ldots-\ldots$ & Rhazisidine & 93. \\
\hline [2] & - . - . - - - - - & Strictanine & 94. \\
\hline [2] & 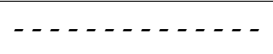 & Strictibine & 95. \\
\hline [2] & 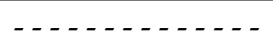 & Stricticine & 96. \\
\hline [2] & - - - - - - - - - & Strictimine & 97. \\
\hline [2] & - - - - - - - - - & Strictimidine & 98. \\
\hline [2] & - - - - - - - - - & Strictine & 99. \\
\hline [2] & $-\cdots-1-\cdots-1$ & Tetrahydropresecamine & 100. \\
\hline [4] & 5280343 & Quercetin & 101. \\
\hline [4] & 72281 & Hesperitin & 102. \\
\hline$[4]$ & 5280863 & Kaempferol & 103. \\
\hline [4] & 5280459 & Quercetin-3-rhamnoside & 104. \\
\hline [4] & 5280804 & Isoquercetin & 105. \\
\hline$[4]$ & 5280805 & Rutin & 106. \\
\hline [4] & 5280443 & Apigenin & 107. \\
\hline [4] & 5280445 & Luteolin & 108. \\
\hline [4] & 5280637 & Luteolin-7-glucoside & 109. \\
\hline$[4]$ & 5280442 & Acacetin & 110. \\
\hline$[4]$ & 5280441 & Apigenin-8-C-glucoside & 111. \\
\hline [27] & 41961 & Tetrahydro-2-(12-pentadecynyloxy)-2H-pyran & 112. \\
\hline [27] & 14276 & Azocine, octahydro- & 113. \\
\hline [27] & 138546 & 8-Azabicyclo[3.2.1]oct-2-ene & 114. \\
\hline [27] & 580053 & 2-Amino-6-methoxypyridine & 115. \\
\hline$[27]$ & 573816 & 4(1H)-Pyridinone, 2,3-dihydro-1-methyl- & 116. \\
\hline [27] & 23494 & Tetratetracontane & 117. \\
\hline [27] & 19901 & Pyridine, 3-ethyl-5-methyl- & 118. \\
\hline$[27]$ & 61038 & 1,3-Propanediol, 2-butyl-2-ethyl- & 119. \\
\hline [27] & 86541 & Nonane, 4,5-dimethyl- & 120. \\
\hline
\end{tabular}


Table 2. Cont.

\begin{tabular}{|c|c|c|c|}
\hline Ref & CID & Compounds & \\
\hline [27] & 11006 & Hexadecane & 121. \\
\hline [27] & 545627 & Dodecane, 4,6-dimethyl- & 122. \\
\hline [27] & 20282 & Dodecane, 1-iodo- & 123. \\
\hline [27] & 520211 & 1-(3-Aminopropyl)-2-pipecoline & 124. \\
\hline [27] & 545941 & 2-Isopropyl-5-methyl-1-heptanol & 125. \\
\hline [27] & 93447 & Dodecane, 2,7,10-trimethyl- & 126. \\
\hline [27] & 285814 & 4,4'-Isopropylidenebis(3-methyl-2-isoxazolin-5-one) & 127. \\
\hline [27] & 541883 & Cyclohexanamine, N-methyl-n-propyl- & 128. \\
\hline [27] & 587705 & Acetamide, 2-(3-hydroxy-8-aza-bicyclo[3.2.1]oct-8-yl)-N-(2,4,6-trimethylphenyl)- & 129. \\
\hline [27] & 73559 & 1H-Isoindole-1,3(2H)-dione, hexahydro- & 130. \\
\hline [27] & 11636 & Heptacosane & 131. \\
\hline [27] & 545611 & Decane, 2,3,5,8-tetramethyl- & 132. \\
\hline [27] & 14536 & Quinoline, 2,4-dimethyl- & 133. \\
\hline [27] & 520709 & Eicosane, 1-iodo- & 134. \\
\hline [27] & 7311 & 2,4-Di-tert-butylphenol & 135. \\
\hline [27] & 14845381 & ((8R,8aS)-8-Isopropyl-5-methyl-3,4,6,7,8,8a-hexahydronaphthalen-2-yl)methanol & 136. \\
\hline [27] & 33865 & 11-Methyldodecanol & 137. \\
\hline [27] & 95337 & 1-Decanol, 2-hexyl- & 138. \\
\hline [27] & 549960 & Cyclohexane, 1-ethyl-2-propyl- & 139. \\
\hline [27] & 67043 & 1-Naphthalenamine, N-ethyl- & 140. \\
\hline [27] & 95997 & $3^{\prime}, 5^{\prime}$-Dimethoxyacetophenone & 141. \\
\hline [27] & 3518 & Guanethidine & 142. \\
\hline [27] & 41209 & Heptadecane, 2,6,10,15-tetramethyl- & 143. \\
\hline [27] & 598127 & Pyridine, 5-methyl-4-phenyl- & 144. \\
\hline [27] & 5281520 & Humulene & 145. \\
\hline [27] & 12798926 & 2-Bromotetradecane & 146. \\
\hline [27] & 20831 & 2-Tetradecanol & 147. \\
\hline [27] & 91693137 & Carbonic acid, eicosyl vinyl ester & 148. \\
\hline [27] & 543807 & 1,7-Dimethyl-4-(1-methylethyl)cyclodecane & 149. \\
\hline [27] & 542202 & Methoxyacetic acid, 2-pentadecyl ester & 150. \\
\hline [27] & 9603606 & Thiourea, (5,5-dimethyl-3-oxo-5,6-dihydropyrrolo[2,1-a]isoquinolin-2-ylidene)- & 151. \\
\hline [27] & 581546 & Sydnone, 3-(2-naphthyl)- & 152. \\
\hline [27] & 152961 & Ethanone, 2-chloro-1H-indol-1-yl- & 153. \\
\hline [27] & 5357283 & 2-Propenoic acid, 3-(4-hydroxy-3-methoxyphenyl)-,methyl ester & 154. \\
\hline [27] & 598515 & 1H-Indole, 4-(3-methyl-2-butenyl)- & 155. \\
\hline [27] & 22833370 & 1,2-Benzenedicarboxylic acid, bis(2-ethylpropyl)ester & 156. \\
\hline [27] & 2936295 & $\begin{array}{l}\text { Pyrrolidine-2,5-dione, } \\
\text { 1-(3-chlorophenyl)-3-(4-phenyl-3,6-dihydro-2H-pyridin-1-yl)- }\end{array}$ & 157. \\
\hline [27] & 42647321 & (6-methylquinolin-2-yl)methanamine & 158. \\
\hline [27] & 8181 & Hexadecanoic acid, methyl ester & 159. \\
\hline [27] & 598481 & 1,7-Dimethylene-2,3-dimethylindole & 160. \\
\hline
\end{tabular}


Table 2. Cont.

\begin{tabular}{|c|c|c|c|}
\hline Ref & CID & Compounds & \\
\hline [27] & 62603 & Benzenepropanoic acid, 3,5-bis(1,1-dimethylethyl)-4-hydroxy-, methyl ester & 161. \\
\hline [27] & 58527531 & Isobutyl tetradecyl ether & 162. \\
\hline [27] & 5364506 & trans-13-Octadecenoic acid, methyl ester & 163. \\
\hline [27] & 5364509 & 9-Octadecenoic acid (Z)-, methyl ester & 164. \\
\hline [27] & 8201 & Methyl stearate & 165. \\
\hline [27] & 5280450 & 9,12-Octadecadienoic acid (Z,Z)- & 166. \\
\hline [27] & 614424 & 3-Amino-5-chloro-benzofuran-2-carboxylic acid methyl ester & 167. \\
\hline [27] & 545593 & Heptacosane, 1-chloro- & 168. \\
\hline [27] & 5376940 & O-(2,4-Dinitrostyryl)-phenol & 169. \\
\hline [27] & 9548854 & Aspidofractinine & 170. \\
\hline [27] & 91693138 & Carbonic acid, octadecyl vinyl ester & 171. \\
\hline [27] & 292723 & Heptadecane, 8-methyl- & 172. \\
\hline [27] & 14259 & Eicosanoic acid, methyl ester & 173. \\
\hline [27] & 292285 & Octadecane, 3-ethyl-5-(2-ethylbutyl)- & 174. \\
\hline [27] & 76958 & Oxiranedodecanoic acid, 3-octyl-, cis- & 175. \\
\hline [27] & 91712839 & Fumaric acid, monoamide, N,N-dimethyl-, 1-naphthyl ester & 176. \\
\hline [27] & 624530 & .beta.-Hydroxyquebrachamine & 177. \\
\hline [27] & 7641 & Hexanedioic acid, bis(2-ethylhexyl) ester & 178. \\
\hline [27] & 594104 & 5,8-Dimethylquinoxaline & 179. \\
\hline [27] & 580937 & Acetic acid, 6-morpholin-4-yl-9-oxobicyclo[3.3.1]non-3-yl ester & 180. \\
\hline [27] & 1969543 & $\begin{array}{l}\text { 1,2,5-Oxadiazole-3-carboxamide, 4-amino-N-[2-[[(3-chlorophenyl) methyl] } \\
\text { amino] ethyl]- }\end{array}$ & 181. \\
\hline [27] & 580956 & $\begin{array}{l}\text { Naphtho[1,2-b]furane-2,8-dione, } \\
\text { 2,3,3a,4,5,5a,8,9b-octahydro-9-methyl-3-(3,3-dimethyl-1-piperidylmethyl)- }\end{array}$ & 182. \\
\hline [27] & 252320 & (+/-)-Uleine & 183. \\
\hline [27] & 5373573 & Apparicine, Nb-methyltetrahydro- & 184. \\
\hline [27] & 112885 & Octadecanoic acid, 3-oxo-, ethyl ester & 185. \\
\hline [27] & 593916 & $\begin{array}{l}\text { 2,20-Cyclo-8,9-secoaspidospermidine, 3-methyl-, } \\
\text { (2.alpha.,3.beta.,5.alpha.,12.beta.,19.alpha.,20R)- }\end{array}$ & 186. \\
\hline [27] & 579942 & 1-Methyl-16-methoxyaspidospermidin-4-one & 187. \\
\hline [27] & 58184953 & 12H-benzo[b]phenoxazine, 12-methyl- & 188. \\
\hline [27] & 20619411 & Methyl 8-methyl-nonanoate & 189. \\
\hline [27] & 610181 & 2-Methyl-7-phenylindole & 190. \\
\hline [27] & 619344 & Eburnamenin-14-ol, 14,15-dihydro-, (14.beta.)- & 191. \\
\hline [27] & 91712719 & 1-Alanine, n-propargyloxycarbonyl-, ethyl ester & 192. \\
\hline [27] & 10949 & 2,4-Diamino-6-methyl-1,3,5-triazine & 193. \\
\hline [27] & 620161 & Indolo[2,3-a]uinolizine-4(12H)-one, 1,2,3,6,7,12b-hexahydro-3,12b-dimethyl- & 194. \\
\hline [27] & 580315 & Aspidospermidine, 1-ethyl- & 195. \\
\hline [27] & 201188 & Vincaminol & 196. \\
\hline [27] & 425980 & Cleavamine & 197. \\
\hline [27] & 91719594 & Phthalic acid, 2-ethylbutyl nonyl ester & 198. \\
\hline
\end{tabular}


Table 2. Cont.

\begin{tabular}{|c|c|c|c|}
\hline Ref & CID & Compounds & \\
\hline [27] & 590836 & Phthalic acid, bis(7-methyloctyl) ester & 199. \\
\hline [27] & 638072 & Squalene & 200. \\
\hline [27] & 71204 & Apovincamine & 201. \\
\hline [31] & 15376 & Vincamine & 202. \\
\hline [31] & 64971 & Betulinic acid & 203. \\
\hline [31] & 382831 & Pomolic acid & 204. \\
\hline [31] & 73659 & Maslinic acid & 205. \\
\hline [31] & 21676297 & Kaempferol rhamnoside rutinoside & 206. \\
\hline [31] & 64945 & Ursolic acid & 207. \\
\hline [31] & 12313704 & Oleanolic acid & 208. \\
\hline [31] & 8969 & Yohimbine & 209. \\
\hline [32] & 998 & Phenylacetaldehyde & 210. \\
\hline [32] & 28111 & (Dimethylamino)methylene malononitrile & 211. \\
\hline [32] & 5283356 & Trans-2-Undecenal & 212. \\
\hline [32] & 12266719 & Dihydrocitronellal & 213. \\
\hline [32] & 62321 & Linalyl butyrate & 214. \\
\hline [32] & 439507 & D-Allose & 215. \\
\hline [32] & 822800 & 1-(3,4-Dimethoxyphenyl) ethanone & 216. \\
\hline [32] & 543855 & 2,2-Tricosenoic acid & 217. \\
\hline [32] & 2537 & Camphor & 218. \\
\hline [32] & 3520 & Guanidine & 219. \\
\hline [33] & 11005 & Myristic acid & 220. \\
\hline [33] & 44256490 & Pentadecylic acid & 221. \\
\hline [33] & 985 & Palmitic acid & 222. \\
\hline [33] & 44256491 & Margaric acid & 223. \\
\hline [33] & 5281 & Stearic acid & 224. \\
\hline [33] & 10467 & Arachidic acid & 225. \\
\hline [33] & 8215 & Behenic acid & 226. \\
\hline [33] & 17085 & Tricosylic acid & 227. \\
\hline [33] & 11197 & Lignoceric acid & 228. \\
\hline [33] & 445638 & Palmitoleic acid & 229. \\
\hline [33] & 445639 & Oleic acid & 230. \\
\hline [33] & 5282761 & Vaccenic acid & 231. \\
\hline [33] & 5282768 & Gondoic acid & 232. \\
\hline [33] & 5281116 & Erucic acid & 233. \\
\hline [33] & 5280450 & Linolic acid & 234. \\
\hline [33] & 5280934 & Linolenic acid & 235. \\
\hline [34] & 441975 & Ajmalicine & 236. \\
\hline [34] & 100004 & Tubotaiwine & 237. \\
\hline [34] & 10314057 & Akuammicine & 238. \\
\hline
\end{tabular}


Table 2. Cont.

\begin{tabular}{|c|c|c|c|}
\hline Ref & CID & Compounds & \\
\hline [34] & 101688177 & Fluorocarpamine & 239. \\
\hline [34] & 624111 & Decarbomethoxytabersonine & 240. \\
\hline [34] & 73391 & Serpentine & 241. \\
\hline [35] & 334274 & Tetrahydrosecamine diol & 242. \\
\hline [36] & $-\ldots-\ldots-\ldots$ & Epi-rhazyaminine & 243. \\
\hline [36] & - - - - - - - - - & 20-epi-sitsirikine & 244. \\
\hline [37] & 85779 & 2-Hexadecanol & 245. \\
\hline [37] & 8973 & 3-O-Methyl-d-glucose & 246. \\
\hline
\end{tabular}

Table 3. Number of $R$. stricta compounds in each group.

\begin{tabular}{cc}
\hline Groups & No. \\
\hline Alkaloid & 118 \\
Fatty acid & 20 \\
Flavonoid & 12 \\
Terpenes & 9 \\
Sterol & 2 \\
Peptides & 1 \\
Others & 85 \\
\hline
\end{tabular}

\section{Toxicity Studies of Rhazya Stricta}

\subsection{Toxicity In Vivo Experiments}

Rhazya stricta leaf was evaluated for its toxicity to Najdi lambs. After oral administration $(1 \mathrm{~g} / \mathrm{kg} / \mathrm{d})$, body weight loss, ruminal, diarrhea, breathlessness, and hind limb weakness were observed. Kidney disease, pulmonary edema, internal bleeding and lung damage, lymphocytes in essential organs, and cardiac vessel congestion were linked to increases in serum AST and LDH, increased bilirubin and urea elevated levels, decreased protein content, albumin, and calcium levels, and leucopenia and anemia [38]. Adult albino rats received intraperitoneal administration $(15 \mathrm{mg} / \mathrm{kg}$ body weight) of $R$. stricta extract that significantly decreased their total number of white blood cells. The following day after injection, there was a substantial drop, and within three days, there was a $50-60 \%$ reduction. The blood cell count recovered to normal after 7-10 days. In dogs, an intravenous dose of the extract $(80 \mathrm{mg} / \mathrm{kg})$ resulted in acute salivation and rigor, followed by respiratory depression, convulsions, and fatality within $15 \mathrm{~min}$ [39]. The LD50 of $R$. stricta extract was determined to be $(16.0 \mathrm{~g} / \mathrm{kg})$ when given orally to mice. At the relatively increased doses used, the plant extract did not cause death and did not appear to be toxic [10]. Another research evaluated the influence of $R$. stricta extract on the growth of rat fetuses. The extract concentration (0.5-2 g/ kg/day) for 3 days in pregnancy had no significant influence on abnormalities. Except for a generalized reduction of growth, no skeletal abnormalities were detected. Increased dosages ( 5 or $8 \mathrm{~g} / \mathrm{kg} /$ day for 3 days) decreased the percentage of viable fetuses and affected placental development, potentially contributing to the reported intrauterine development abnormalities and fetal mortality [40].

\subsection{Genotoxicity and Mutagenicity}

Through the comet assay, significant increases in genotoxicity were observed for $R$. stricta extract at $10 \mathrm{mg} / \mathrm{mL}$ doses at different time points. Mutagenicity was tested by using Ames Salmonella assay. R. stricta was determined not to be mutagenic to Salmonella typhimurium (TA100) and Salmonella typhimurium (TA98) [41]. Saccharomyces cerevisiae suspensions were exposed to increasing amounts of aqueous extract of the R. stricta leaf. The extract was found to have significant lethal and mutagenic activity. As the concentration 
or duration of exposure increased, the survival percentage decreased [42]. Another study tested three $R$. stricta extracts by administering them to rats via oral gavage independently, and the three extracts were whole aqueous, alkaloid, and nonalkaloid. The results suggested that whole aqueous and alkaloid extracts of $R$. stricta altered the genomic randomly amplified polymorphic DNA profile, induced significant DNA damage, increased the formation of micronuclei, induced chromosomal aberrations, and decreased the mitotic index [43].

\section{Pharmacological Activities of R. stricta}

\subsection{Antibacterial Activity}

In vitro, R. stricta leaf and fruit extract demonstrated antibacterial activity against Staphlococcus aureus, Escherichia coli, Pseudomonasaeruginosa, Bacillus subtilis, Streptococcus pyogenes, and Salmonella typhi [44]. R. stricta leaf extract demonstrated a control of bacterial growth on locally isolated meningococcal strains that increased with concentration and treatment time [45]. Chloroformic and methanolic extracts of R. stricta roots exhibited antimicrobial activity toward B. subtilis, E. coli, S. aureus, and P. aeruginosa. Tetrahydrosecamine was purified from the plant and demonstrated a wide range of antibacterial activity (effective toward all bacteria except E. coli; MIC values ranged from 0.1 to $5.0 \mathrm{mg} / \mathrm{mL}$ ). Another active substance, strictanol, was also shown to be effective against P. aeruginosa and E. coli (MIC $0.5 \mathrm{mg} / \mathrm{mL}$ for both microbes) [46]. The Ag nanoparticles synthesized using silver nitrate and methanol root extract of R. stricta showed improved antibacterial activity against B. subtilis and E. coli [47]. At low concentrations, the tested R. stricta extract mixed with Ag nanoparticles inhibited the growth of several pathogenic bacteria, including Klebsiella pneumoniae, B. subtilis, and S. typhi [31]. The antibacterial activity of five R. stricta leaf extracts at various concentrations was examined against a board of gram-negative and gram-positive bacteria. R. stricta organic alkaloid extract was most effective against E. coli and methicillin-resistant Staphylococcus aureus (MRSA), resulting in the disruption of cell membranes [48]. Acetone and the methanolic extract of R. stricta leaves demonstrated antibacterial activity against Propionibacterium acnes at a (50 mg/mL) minimum inhibitory concentration and zone of inhibition $25.6 \pm 1.94 \mathrm{~mm}$ [49]. The biogenic Au nanoparticles and R. stricta extract degraded the membrane of E. coli (MIC1 $425.0 \mathrm{mg} / \mathrm{mL}$ ) and B. subtilis $(50.0 \mathrm{mg} / \mathrm{mL})$, and stimulated the production of reactive oxygen species, resulting in the death of microbial cells [50]. R. stricta extract suppressed methicillin-resistant Staphylococcus aureus (MRSA) growth, with zones of inhibition extending from 6 to $19 \mathrm{~mm}$, and transmission electron microscopy demonstrated that the extract alters MRSA bacteria cellular architecture [51].

\subsection{Antifungal Activity}

R. stricta chloroformic and methanolic root fractions demonstrated antifungal activities against Aspergillus terreus, Aspergillus flavus, and Candida albicans [46]. Another study revealed that fractionated $R$. stricta methanol and chloroform samples showed antifungal activity against Trichophyton longifusis, C. albicans, A. flavus, and Fusarium solani [52].

\subsection{Antioxidant Activity}

At some doses, $R$. stricta extract exhibits antioxidant effects in rats by increasing glutathione levels and decreasing lipid peroxidation [53]. In comparison to the tocopherol drug and the synthetic antioxidant butylated hydroxyanisole, $R$. stricta methanolic extract was a significant source of natural antioxidants with high free radical scavenging and anion radical scavenging potentials [54]. Significant lipoxygenase and acetylcholinesterase inhibitory activity were observed by the ethanolic extract of $R$. stricta fruit [44]. To determine the impact of the climate conditions on $R$. stricta, the plant leaves collected from Riyadh and the western region were extracted. Both extracts exhibited antioxidant activity, with significant superior performance to $R$. stricta leaves collected from the western region by six evaluation of superoxide radical scavenging and scavenging of hydrogen peroxide 
levels [4]. The antioxidant activity of root fractions of $R$. stricta was determined using a variety of antioxidant assays. The fractions obtained by solvent-solvent extraction of $R$. stricta root raw extract exhibited remarkable free radical scavenging activity, with an IC50 of $400-776 \mathrm{~g} / \mathrm{mL}$ [55].

\subsection{Anticancer Activity}

Tetrahydrosecamine diol, which was identified in $R$. stricta, possesses remarkable anticancer activity in vitro against $\mathrm{KB}$ carcinoma of the nasopharynx with an $\mathrm{ED}_{50} 0.0038 \mu \mathrm{g} / \mathrm{mL}$ [56]. Rhazinilam, which mimics taxol cellular activity by suppressing both microtubule assembly and disassembly in vitro, supported the formation of abnormal tubulin spirals and resulted in the formation of microtubule bundles, multiple asters, and microtubule constancy at low temperatures. In vitro, rhazinilam was cytotoxic to a wide range of cancer cell lines at low micromolar concentrations, but it displayed no activity in vivo [57]. R. stricta ethanol extract induced apoptosis in breast cancer cells by inhibiting cellular growth and colony formation, stating that it may be a beneficial chemo-preventive or drug product in the treatment of breast cancer [58]. Treatment of MDA-MB-231 cells with $R$. stricta fruit ethyl acetate fraction increased p53, Bax, and caspase 3/7 expression and activation. A cell migration scratch assay indicated that the extract at non-cytotoxic concentrations inhibited the highly invasive MDA-MB-231 cell lines migration. Additionally, RT-PCR analysis revealed significantly decreased (MMP-2) and (MMP-9) expression, both of which play a critical role in breast cancer metastasis. Breast tissue histological assessments in experimental animals revealed a slight improvement in tissue treated with fruit ethyl acetate fraction [32]. On HepG2 and Caco cells, the ethanol extract of R. stricta was highly effective (IC50 values of $25 \mu \mathrm{g} / \mathrm{mL}$ and $35 \mu \mathrm{g} / \mathrm{mL}$, respectively) [59]. Additionally, an in vivo study established experimental evidence by measuring serum liver enzymes and the histopathological alteration of liver tissue for the methanol extract of $R$. stricta aerial parts antitumor efficacy against hepatocellular carcinoma. This effect may be a result of the compound's hepatoprotective properties, antiproliferative activity, and antiangiogenic potential [60]. The crude alkaloid extract of $R$. stricta significantly induced apoptosis in pancreatic cancer cells with IC50 (78.77 and $41.4 \mu \mathrm{g} / \mathrm{mL})$ on PANC-1 and AsPC-1 cell lines [61]. The ethanol extract of $R$. stricta leaves suppressed colony formation development in HepG2 cells and significantly restricted cell cycle in the G2/M phase 12 and $48 \mathrm{~h}$ following administration, as well as substantial limitation at the G1/S phase after $24 \mathrm{~h}$. This finding supports the use of $R$. stricta as a novel anticancer agent in the treatment of hepatocellular carcinoma [62]. The compounds Epi-rhazyaminine, 20-epi-sitsirikine, eburenine, strictamine, (16R)-Eisositsirikine, antirhine, and strictanol were identified and tested using the MTT assay targeting three types of cancer cells (HCT-116, PC-3, and HepG2) as well as a single kind of normal cell (VERO). The phytochemicals studied had a weak cytotoxic effect on the three cancer cell lines [36]. During 24 and $48 \mathrm{~h}$ period assays, R. stricta nanoparticles had a substantial inhibitory impact on Hep G-2 cell viability at concentrations of 100 and $500 \mathrm{~g} / \mathrm{mL}$ [63].

\subsection{Antidiabetic Activity}

$R$. stricta water extract showed no noticeable impact on a glucose concentration introduced orally to rats with and without diabetes. Frequent treatment of $R$. stricta in a water supply had no effect on the glucose homeostasis measures investigated (plasma glucose, body mass, feed and fluid intake, and blood fructosamine) during a 37-day period in either the normal or diabetic stage of this study [64]. The acute administration of the lyophilized $R$. stricta extract to rats at a dosage of $4 \mathrm{~g} / \mathrm{kg}$ resulted in an important increase in insulin concentration. $R$. stricta at a dosage of $8 \mathrm{~g} / \mathrm{kg}$ significantly decreased plasma glucose concentrations at 0.5 and $1 \mathrm{~h}$ after treatment in streptozotocin-diabetic rats loaded orally with glucose $(1 \mathrm{~g} / \mathrm{kg})$. Chronic administration with a lyophilized extract of $R$. stricta to mice and rats for 28 days did not impact plasma, glucose, or insulin concentrations or on any of the hematological or biochemical parameters examined [65]. R. stricta extract was 
administered orally to diabetic rats at dosages of $(0.5,20$, and $4.0 \mathrm{~g} / \mathrm{kg})$ and the glucose level was significantly lowered $1 \mathrm{~h}(2$ and $4 \mathrm{~g} / \mathrm{kg})$ and $2 \mathrm{~h}(4 \mathrm{~g} / \mathrm{kg})$ after the extract was administered. This was followed by substantial increases in insulin concentrations 1,2 , and $4 \mathrm{~h}$ after the extract was administered at dosages of $(2$ and $4 \mathrm{~g} / \mathrm{kg}$ ). Combined administration of hyperglycemic rats with the leaf extract $(0.5,20$ and $5.0 \mathrm{~g} / \mathrm{kg})$ and glibenclamide $(5.0 \mathrm{mg} / \mathrm{kg})$ substantially increased the effects of the extract or glibenclamide on glucose, insulin, and glucagon when used alone. When the leaf extract was administered at dosages of $(0.5,2$, and $4 \mathrm{~g} / \mathrm{kg})$ daily for six successive days, the glucose level decreased by about 6 , 8 , and $30 \%$, respectively [66]. The effects of $R$. stricta extract on adiponectin concentrations could be beneficial in the treatment of diabetes by increasing the adiponectin level [67]. $R$. stricta root extract demonstrated significant antidiabetic activity by inhibiting Dipeptidyl peptidase-IV (up to $61 \%$ ) and $\beta$-secretase (up to $83 \%$ ) enzymes, resulting in an increase in glucagon-like peptide- 1 secretion [27]. The ethyl acetate fraction of $R$. stricta is most effective at lowering blood glucose amounts in fasting and random conditions, and the lowering of blood glucose levels was similar to that of Glucophage, a basic antidiabetic drug [68].

\subsection{Other Pharmacological Activities}

The lyophilized extract of $R$. stricta $(5-100 \mathrm{mg} / \mathrm{kg}$ ) concentrations had a variable impact on heart rate and a dose-dependent reduction in mean blood pressure in urethaneanaesthetized rats [65]. The potential that part of the R. stricta extract stated therapeutic effects are related to its immunomodulatory capacity was explored in one experiment using ex vivo generation of macrophage-derived cytokines in mice. Every mouse was treated twice weekly with an alkaloidal portion of $R$. stricta $(0.5$ and $1.0 \mathrm{mg} /$ individual). Peritoneal cells were extracted, grown, and tested for IL-1a and TNF using an enzymelinked immunosorbent assay (ELISA). R. stricta's alkaloidal portion considerably enhanced the secretion of these two proinflammatory cytokines [69]. When compared to other UAE medicinal plants, $R$. stricta demonstrated the strongest ability to relax smooth muscles, implying that the herb may have antispasmodic capabilities. This appears to corroborate the plant's folk medicinal use in certain regions [3]. The chloroform stem extract of $R$. stricta can stimulate early neuronal differentiation in stem cells and may possess a potential therapeutic agent for neurodegenerative diseases [70]. The methanol extract of $R$. stricta significantly reduced the degree and frequency of diarrhea in rats caused by castor oil. Moreover, $R$. stricta extract significantly reduced castor oil-induced intestinal transit by $24.44 \%$ at a dosage of $(250 \mathrm{mg} / \mathrm{kg})$ and $58.88 \%$ at a dosage of $(500 \mathrm{mg} / \mathrm{kg})$ [71]. The immunomodulatory impact of $R$. stricta methanol extract was investigated by giving it to broiler chicks in their drinking water for two weeks before they were challenged with sheep erythrocytes. Significant increases in phagocytic activity, lymphocyte proliferation, and percentages of circulating lymphocytes were detected, indicating an improvement in cellular immunity. Significant increases in the serum levels of total antibodies of the IgM and IgG isotypes were also seen, indicating an improved humoral response [37].

\section{Biological Activities}

The raw extract of $R$. stricta was found to be larvicidal and inhibited growth (8-36\%) with increasing doses (200-1000 ppm) in Aedes aegypti fourth instar larvae [72]. By impairing membrane function and photosynthetic ability, the leaf extract of $R$. stricta inhibits the growth and metabolic activity of Salsola villosa [73]. The high growth rate of $R$. stricta, its resistance to heavy toxic metals, and its capacity to absorb and concentrate metals inside the plant all support its application in phytoremediation [74]. With an increase in the concentrations of the leaf extract of the medicinal plant $R$. stricta, the mortality and repellency of Rhyzopertha dominica and Trogoderma granarium increased. Thus, $R$. stricta may be a useful ingredient in an effective pest control system designed to combat stored grain pests [75]. Dry powdered leaves or succulent shoots of $R$. stricta $(30 \mathrm{~g} / \mathrm{kg}$ of soil), thoroughly mixed with soil 20 days before transplanting, may act as an effective control 
method against bacterial wilt [76]. R. stricta methanolic extract inhibited seed germination of Phalaris minor, Chenopodium album, and Rumex dentatus by percentages of $43 \%, 47 \%$, and $42 \%$, respectively, in soil. $R$. stricta demonstrated promising allelopathic activity [77]. Water extract of $R$. stricta was applied at concentrations ranging from 100 to 500 parts per million, inhibiting the growth by reducing hatchability of eggs and causing the death of Culex pipiens mosquitoes [78]. R. stricta extract also demonstrated nematocidal activity against the nematode Meloidogyne javanica at a concentration of $100 \mathrm{ppm}$ [79]. After $48 \mathrm{~h}$ of incubation, $100 \mathrm{mg} / \mathrm{mL}$ gold nanoparticles of $R$. stricta aqueous extract inhibited the growth of intra-THP-1 amastigotes (IC50: $1443 \mathrm{mg} / \mathrm{mL}$ ) [50]. R. stricta extracts demonstrated the ability to suppress nutsedge density, length, and weight (fresh and dry) of the root and shoot [80]. R. stricta extract resulted in (91\%) mortality in Culex pipiens by decreasing the expression level of acetylcholinesterase and glutathione S-transferase [81]. The ZnO nanoparticles and leaf extract of Rhazya stricta were revealed to be effective antimalarial agents at a 50\% inhibitory concentration (IC50: $3.41 \mathrm{~g} / \mathrm{mL}$ ) [82]. When injected into the blood stream, an aqueous extract of the stems and roots was somewhat poisonous to American cockroaches but had no impact on German cockroaches or milkweed bugs [83].

\section{Conclusions}

For over 50 years, phytochemicals, pharmacological, and biological activities of $R$. stricta whole extract were the focus of attention in the Middle East and the South of Asia. $R$. stricta extract has been found to be toxic in animal models, as well as genotoxic and mutagenic in microorganism models, according to several studies. The phytochemistry profile of $R$. stricta contains a unique alkaloid content that has been isolated and identified significantly, and we found that the non-alkaloid contents need more investigation. $R$. stricta extract has shown pharmacological activity such as antimicrobial, anticancer, antidiabetic, and antioxidant activities, as well as biological activity such as insecticide, allelopathic, and soil remediation activities. Some pharmacological aspects, such as the antiviral activity of the plant extract have not been examined yet. Despite plenty of studies investigating $R$. stricta activity, only a few studies investigated the activity of its unique phytochemicals individually, so the advancement of research on the R. stricta plant should be moving from the whole extract level to the phytochemical levels.

Author Contributions: Writing—original draft preparation, A.A. Reviewing and editing, N.A.B., T.A.B., A.A.A. All authors have read and agreed to the published version of the manuscript.

Funding: This research received no external funding.

Institutional Review Board Statement: Not applicable.

Informed Consent Statement: Not applicable.

Data Availability Statement: All data included in the main text.

Conflicts of Interest: The authors declare no conflict of interest.

\section{References}

1. Park, S.; Ruhlman, T.A.; Sabir, J.S.; Mutwakil, M.H.; Baeshen, M.N.; Sabir, M.J.; Baeshen, N.A.; Jansen, R.K. Complete sequences of organelle genomes from the medicinal plant Rhazya stricta (Apocynaceae) and contrasting patterns of mitochondrial genome evolution across asterids. BMC Genom. 2014, 15, 405. [CrossRef] [PubMed]

2. Marwat, S.K.; Usman, K.; Shah, S.S.; Anwar, N.; Ullah, I. A review of phytochemistry, bioactivities and ethno medicinal uses of Rhazya stricta Decsne (Apocynaceae). Afr. J. Microbiol. Res. 2012, 6, 1629-1641.

3. Ali, B.H.; Al-Qarawi, A.A.; Bashir, A.K.; Tanira, M.O. Phytochemistry, pharmacology and toxicity of Rhazya stricta Decne: A review. Phytother. Res. Int. J. Devoted Pharmacol. Toxicol. Eval. Nat. Prod. Deriv. 2000, 14, 229-234.

4. Bukhari, N.A.; Al-Otaibi, R.A.; Ibhrahim, M.M. Phytochemical and taxonomic evaluation of Rhazya stricta in Saudi Arabia. Saudi J. Biol. Sci. 2017, 24, 1513-1521. [CrossRef]

5. Roberts, M.F.; Wink, M. Biochemistry, ecology, and medicinal applications. In Alkaloids; Plenum Press: New York, NY, USA, 1998; pp. 1-7.

6. Gilani, S.A.; Kikuchi, A.; Shinwari, Z.K.; Khattak, Z.I.; Watanabe, K.N. Phytochemical, pharmacological and ethnobotanical studies of Rhazya stricta Decne. Phytother. Res. PTR 2007, 21, 301-307. [CrossRef] 
7. Rhazya stricta Decne. in GBIF Secretariat (2021). GBIF Backbone Taxonomy. Available online: https://www.gbif.org/dataset/d7 dddbf4-2cf0-4f39-9b2a-bb099caae36c (accessed on 2 October 2021). [CrossRef]

8. Bhadane, B.S.; Patil, M.P.; Maheshwari, V.L.; Patil, R. Ethnopharmacology, phytochemistry, and biotechnological advances of family Apocynaceae: A review. Phytother. Res. 2018, 32, 1181-1210. [CrossRef]

9. Ali, B.H.; Bashir, A.K.; Tanira, M.O.M. The effect of Rhazya stricta Decne, a traditional medicinal plant, on the forced swimming test in rats. Pharmacol. Biochem. Behav. 1998, 59, 547-550. [CrossRef]

10. Ali, B.H.; Bashir, A.K.; Banna, N.R.; Tanira, M.O.M. Central Nervous System Activity of Rhazya Stricta (Decne) In Mice. Clin. Exp. Pharmacol. Physiol. 1995, 22, 248-253. [CrossRef]

11. Ghazanfar, S.A.; Al-Al-Sabahi, A.M. Medicinal plants of Northern and Central Oman (Arabia). Econ. Bot. 1993, 47, 89-98. [CrossRef]

12. Lace, J.H.; Hemsley, W.B. A Sketch of the Vegetation of British Baluchistan, with Descriptions of New Species. J. Linn. Soc. Lond. Bot. 1891, 28, 288-327. [CrossRef]

13. Fatima, K. Isolation and Structure Elucidation of Alkaloids from "Rhazya stricta". Ph.D Thesis, H.E.J. Research Institute of Chemistry/University of Karachi, Karachi, Pakistan, 1980; pp. 1-123.

14. Rehman, H.U. Isolation and Structural Studies on Chemical Constituents of Rhazya Stricta and Related Medicinal Plants. Ph.D. Thesis, HEJ Research Institute of Chemistry, ICCBS, University of Karachi, Karachi, Pakistan, 1987.

15. Qureshi, M.M. Isolation and Structural Studies on the Chemical Constituents of Rhazya Stricta 1987, Alstonia Macrophylla and Related Medicinal Plants. Ph.D. Thesis, University of Karachi, Karachi, Pakistan, 1991.

16. Habbib-ur-Rehman, A.U.R. Alkaloids of the leaves of Rhazya stricta. Fitoterapia 1996, 37, 145-148.

17. Banerji, A.; Majumder, P.; Chatterjee, A. Occurrence of geissoschizine and other minor biogenetically related alkaloids in Rhazya stricta. Phytochemistry 1970, 9, 1491-1493. [CrossRef]

18. Rahman, A.U.; Zaman, K. 1,2-dehydroaspidospermidine-N-oxide, an alkaloid from Rhazya stricta. Phytochemistry 1986, 25, 1779-1780. [CrossRef]

19. Rahman, A.U.; Zaman, K.; Rehman, H.U.; Malik, S. Studies on the Alkaloids of Rhazya stricta. J. Nat. Prod. 1986, 49, 1138-1139. [CrossRef]

20. Mukhopadhyay, S.; El-Sayed, A.; Handy, G.A.; Cordell, G.A. Catharanthus Alkaloids XXXVII. 16-Epi-Z-Isositsirikine, a Monomeric Indole Alkaloid with Antineoplastic Activity from Catharanthus roseus and Rhazya stricta. J. Nat. Prod. 1983, 46, 409-413. [CrossRef]

21. Khanum, S. Isolation and Structural studies on alkaloids of Rhyazya stricta and synthetic studies in the field of pyridyl indole, Institute HEJ Res. Chem. Uni. Karachi 1986. [CrossRef]

22. Ahmad, Y.; Fatima, K.; Le Quesne, P.W.; Rahman, A.U. Further alkaloidal constituents of the leaves of Rhazya stricta. Phytochemistry 1983, 22, 1017-1019. [CrossRef]

23. Mariee, N.K.; Khalil, A.A.; Nasser, A.A.; Al-Hiti, M.M.; Ali, W.M. Isolation of the Antimicrobial Alkaloid Stemmadenine from Iraqi Rhazya stricta. J. Nat. Prod. 1988, 51, 186-187. [CrossRef]

24. Saeed, S.A.; Simjee, R.U.; Mahmood, F.; Sultana, N. Rhazimine fromRhazya stricta: A Dual Inhibitor of Arachidonic Acid Metabolism and Platelet Activating Factor-Induced Platelet Aggregation. Planta Med. 1993, 59, 566-567. [CrossRef]

25. Lanjwani, A.H.; Ganghro, A.B.; Khuhawar, T.M.J. Phytochemical analysis and biological activity of diffirent parts of rhazya stricta. Rawal Med. J. 2018, 43, 532-535.

26. Baloch, W.B.; Memon, N.; Rani, M.; Abbasi, A.R.; Khan, S.; Memon, A.; Hassan Imran, A.; Ullah, L. Nutritional composition of Rhazya stricta, A local medicinal plant of Kech Region, Pakistan. Rawat Med. J. 2016, 41, 363-368.

27. Mahmood, R.; Kayani, W.K.; Ahmed, T.; Malik, F.; Hussain, S.; Ashfaq, M.; Ali, H.; Rubnawaz, S.; Green, B.D.; Calderwood, D.; et al. Assessment of antidiabetic potential and phytochemical profiling of Rhazya stricta root extracts. BMC Complement. Med. Ther. 2020, 20, 293. [CrossRef]

28. Bibi, H.; Iqbal, M.; Wahab, H.; Öztürk, M.; Ke, F.; Iqbal, Z.; Khan, M.I.; Alghanem, S.M. Green synthesis of multifunctional carbon coated copper oxide nanosheets and their photocatalytic and antibacterial activities. Sci. Rep. 2021, 11, 10781. [CrossRef]

29. National Center for Biotechnology Information. PubChem Compound Summary. Available online: https://pubchem.ncbi.nlm. nih.gov/ (accessed on 31 May 2021).

30. Obaid, A.Y.; Voleti, S.; Bora, R.S.; Hajrah, N.H.; Omer, A.M.S.; Sabir, J.S.M.; Saini, K.S. Cheminformatics studies to analyze the therapeutic potential of phytochemicals from Rhazya stricta. Chem. Cent. J. 2017, 11, 11. [CrossRef] [PubMed]

31. Aziz, A.T.; Alshehri, M.A.; Alanazi, N.A.; Panneerselvam, C.; Trivedi, S.; Maggi, F.; Sut, S.; Dall'Acqua, S. Phytochemical analysis of Rhazya stricta extract and its use in fabrication of silver nanoparticles effective against mosquito vectors and microbial pathogens. Sci. Total. Environ. 2020, 700, 134443. [CrossRef] [PubMed]

32. Al-Zharani, M.; Nasr, F.A.; AbuTaha, N.; Alqahtani, A.S.; Noman, O.M.; Mubarak, M.; Wadaan, M.A.; Zharani, A. Nasr Apoptotic Induction and Anti-Migratory Effects of Rhazya Stricta Fruit Extracts on a Human Breast Cancer Cell Line. Molecules 2019, 24, 3968. [CrossRef] [PubMed]

33. Nehdi, I.A.; Sbihi, H.M.; Tan, C.P.; Al-Resayes, S.I. Seed oil from Harmal (Rhazya stricta Decne) grown in Riyadh (Saudi Arabia): A potential source of $\delta$-tocopherol. J. Saudi Chem. Soc. 2016, 20, 107-113. [CrossRef]

34. Akhgari, A.; Oksman-Caldentey, K.-M.; Rischer, H. Biotechnology of the medicinal plant Rhazya stricta: A little investigated member of the Apocynaceae family. Biotechnol. Lett. 2017, 39, 829-840. [CrossRef] 
35. Evans, D.A.; Smith, G.F.; Smith, G.N.; Stapleford, K.S.J. Rhazya alkaloids: The secamines, a new group of indole alkaloids. Chem. Commun. 1968, 15, 859-861. [CrossRef]

36. Abdul-Hameed, Z.H.; Alarif, W.M.; Sobhi, T.R.; Abdel-Lateff, A.; Ayyad, S.-E.N.; Badria, F.A.; Saber, J. New cytotoxic indole-type alkaloids obtained from Rhazya stricta leaves. South Afr. J. Bot. 2021, 137, 298-302. [CrossRef]

37. Albarrak, S.M. Antioxidant and immune responses of broiler chickens supplemented with Rhazya stricta extract in drinking water. Veter. World 2021, 14, 1437-1449. [CrossRef]

38. Adam, S.E. Toxicity of Rhazya stricta to sheep. Veter. Hum. Toxicol. 1998, 40, 68-69.

39. Siddiqui, S.; Bukhari, A.Q.S. I-Leucopenic Effect of Rhazya stricta. Nature 1972, 235, 393. [CrossRef]

40. Rasheed, R.A.; Bashir, A.K.; Ali, B.H.; Padmanabhan, R. Effect of Rhazya stricta on the developing rat fetus. Reprod. Toxicol. 1997, 11, 191-199. [CrossRef]

41. Hameed, S.S.; ElAssouli, M.-Z.M.; Alhejin, A.M.; Alam, M.Z.; ElAssouli, S.M.; Filimban, F.Z. Evaluation of genotoxicity and mutagenicity of aqueous extracts of Rhazya stricta Decne. and Thymus vulgaris L. Orient. Pharm. Exp. Med. 2018, 18, 357-363. [CrossRef]

42. Baeshin, N.A.; Twaty, N.; Al-Hebshi, A. Evaluating the genotoxicity of Rhazya stricta leaves extract by the Saccharomyces cerevisiae auxotrophic mutants test. Egyp. J. Nat. Toxi 2005, 2, 87-100.

43. Baeshen, N.A.; Elkady, A.I.; Yaghmoor, S.S.; Al Ashmaoi, H.M.; Kumosani, T.A. Evaluation of the cytotoxicity and genotoxicity of alkaloid-rich and alkaloid-free aqueous extracts of Rhazya stricta leaves. Bothalia J. 2014, 44, 358-371.

44. Sultana, N.; Khalid, A. Phytochemical and enzyme inhibitory studies on indigenous medicinal plantRhazya stricta. Nat. Prod. Res. 2010, 24, 305-314. [CrossRef]

45. Abadi, F.; Abdulaziz, A.; Hadhoud, A.; Baeshin, N.; Qari, S.; Alhejin, A.M. An epidemiological survey and evaluation of the antimicrobial growth effect of Rhazya stricta (Decne) leaves extract on different genotypes of Neisseria meningitides. Egypt $J$. Med. Microbiol. 2011, 20, 77À86.

46. Bashir, A.K.; Abdalla, A.A.; Hassan, E.S.; Wasfi, I.A.; Amiri, M.A.; Crabb, T.A. Alkaloids with antimicrobial activity from the root of Rhyzya stricta Decn. growing in United Arab Emirates. Arab Gulf J. Sci. Res. 1994, 12, 119-131.

47. Shehzad, A.; Qureshi, M.; Jabeen, S.; Ahmad, R.; Alabdalall, A.H.; Aljafary, M.A.; Al-Suhaimi, E. Synthesis, characterization and antibacterial activity of silver nanoparticles using Rhazya stricta. PeerJ 2018, 6, e6086. [CrossRef]

48. Khan, R.; Baeshen, M.N.; Saini, K.S.; Bora, R.S.; Al-Hejin, A.M.; Baeshen, N.A. Antibacterial activities of Rhazya stricta leaf extracts against multidrug-resistant human pathogens. Biotechnol. Biotechnol. Equip. 2016, 30, 1016-1025. [CrossRef]

49. Alnabati, N.A.; Al-Hejin, A.M.; Noor, S.O.; Ahmed, M.M.M.; Abu-Zeid, M.; Mleeh, N.T. The antibacterial activity of four Saudi medicinal plants against clinical isolates of Propionibacterium acnes. Biotechnol. Biotechnol. Equip. 2021, 35, 415-424. [CrossRef]

50. Ahmad, A.; Wei, Y.; Ullah, S.; Shah, S.I.; Nasir, F.; Shah, A.; Iqbal, Z.; Tahir, K.; Khan, U.A.; Yuan, Q. Synthesis of phytochemicalsstabilized gold nanoparticles and their biological activities against bacteria and Leishmania. Microb. Pathog. 2017, 110, 304-312. [CrossRef] [PubMed]

51. Khan, R.; Baeshen, M.N.; Saini, K.S.; Al-Hejin, R.S.B.A.M. Antibacterial Activity of Rhazya stricta Non-alkaloid Extract against Methicillin-Resistant Staphylococcus aureus. Biol. Syst. Open Access 2016, 5, 2. [CrossRef]

52. Khan, S.; Khan, G.M. In vitro antifungal activity of Rhazya stricta. Pak. J. Pharm. Sci. 2007, 20, 279-284. [PubMed]

53. Ali, B.H.; Alqarawi, A.A.; Bashir, A.K.; Tanira, M.O. Antioxidant action of extract of the traditional medicinal plant Rhazya stricta Decne. in rats. Phytother. Res. Int. J. Devoted Pharmacol. Toxicol. Eval. Nat. Prod. Deriv. 2000, 14, 469-471.

54. Iqbal, S.; Bhanger, M.; Akhtar, M.; Anwar, F.; Ahmed, K.R.; Anwer, T. Antioxidant Properties of Methanolic Extracts from Leaves of Rhazya stricta. J. Med. Food 2006, 9, 270-275. [CrossRef]

55. Mahmood, R.; Malik, F.; Shamas, S.; Ahmed, T.; Kausar, M.; Rubnawaz, S.; Ashfaq, M.; Hussain, S.; Green, B.D.; Mirza, B. Pharmacological evaluation of Rhazya stricta root extract. Boletín Latinoam. Y Del Caribe De Plantas Med. Y Aromáticas 2020, 19, 0717-7917.

56. Mukhopadhyay, S.; Handy, G.A.; Funayama, S.; Cordell, G.A. Anticancer Indole Alkaloids of Rhazya stricta. J. Nat. Prod. 1981, 44, 696-700. [CrossRef]

57. Gu, Z.; Zakarian, A. Total Synthesis of Rhazinilam: Axial to Point Chirality Transfer in an Enantiospecific Pd-Catalyzed Transannular Cyclization. Org. Lett. 2010, 12, 4224-4227. [CrossRef]

58. Baeshen, N.A.; Elkady, A.I.; Abuzinadah, O.A.; Mutwakil, M.H. Potential anticancer activity of the medicinal herb, Rhazya stricta, against human breast cancer. Afr. J. Biotechnol. 2012, 11, 8960-8972.

59. El-Awady, M.A.; Awad, N.S.; El-Tarras, A.E. Evaluation of the anticancer activities of pomegranate (Punica granatum) and harmal (Rhazya stricta) plants grown in Saudi arabia. Int. J. Curr. Microbiol. App. Sci. 2015, 4, 1158-1167.

60. Shahat, A.A.; Alsaid, M.S.; Kotob, S.E.; Husseiny, H.A.; Al-Ghamdi, A.A.; Ahmed, H.H. Biochemical and histological evidences for the antitumor potential of Teucrium Oliverianum and Rhazya stricta in chemically-induced hepatocellular carcinoma. Afr. $J$. Tradit. Complement. Altern. Med. 2016, 13, 62. [CrossRef]

61. Shaer, N.A. Can crude alkaloids extract of Rhazya stricta induce apoptosis in pancreatic cancer: In vitro study? Pathophysiology 2019, 26, 97-101. [CrossRef]

62. Al-Dabbagh, B.; Elhaty, I.A.; Al Hrout, A.; Al Sakkaf, R.; El-Awady, R.; Ashraf, S.S.; Amin, A. Antioxidant and anticancer activities of Trigonella foenum-graecum, Cassia acutifolia and Rhazya stricta. BMC Complement. Altern. Med. 2018, 18, 240. [CrossRef] 
63. Al-Abbas, N.; Shaer, N. Apoptotic and Anti-Proliferative Effects of Rhazya Stricta Nanoparticles against Hepatocellular Carcinoma (Hep G-2 And Huh-7) Cell Lines. Mansoura Med. J. 2021, 50, 27-35. [CrossRef]

64. Wasfi, I.; Bashir, A.; Amiri, M.; Abdalla, A. The effect of Rhazya stricta on glucose homeostasis in normal and streptozoticin diabetic rats. J. Ethnopharmacol. 1994, 43, 141-147. [CrossRef]

65. Tanira, M.; Ali, B.; Bashir, A.; Chandranath, I. Some pharmacologic and toxicologic studies on rhazya stricta decne in rats, mice and rabbits. Gen. Pharmacol. Vasc. Syst. 1996, 27, 1261-1267. [CrossRef]

66. Ali, B.H. The Effect on Plasma Glucose, Insulin and Glucagon Levels of Treatment of Diabetic Rats with the Medicinal Plant Rhazya stricta and with Glibenclamide, Alone and in Combination. J. Pharm. Pharmacol. 1997, 49, 1003-1007. [CrossRef]

67. Baeshen, N.; Lari, S.; Al Doghaither, H.A.; Ramadan, H.A. Effect of Rhazya stricta extract on rat adiponectin gene and insulin resistance. J. Am. Sci. 2010, 6, 1237-1245.

68. Ahmed, A.; Asad, M.J.; Ahmad, M.S.; Qureshi, R.; Shah, S.I.; Gul, H.; Gulfraz, M. Antidiabetic and hypolipidemic potential of Rhazya stricta Decne extract and its fractions. Int. Curr. Pharm. J. 2015, 4, 353-361. [CrossRef]

69. Tanira, M.O.; Ali, B.H.; Bashir, A.K.; Stephen, S.; Lukic, M. Rhazya stricta enhances IL-1 and TNF $\alpha$ production by macrophages in mice. FASEB J. 1998, 12, 0892-6638.

70. Alagrafi, F.S.; Alawad, A.O.; AbuTaha, N.M.; Nasr, F.A.; Alhazzaa, O.A.; Alharbi, S.N.; Alkhrayef, M.N.; Hammad, M.; Alhamdan, Z.A.; Alenazi, A.D.; et al. In vitro induction of human embryonal carcinoma differentiation by a crude extract of Rhazya stricta. BMC Complement. Altern. Med. 2017, 17, 342. [CrossRef]

71. Al-Harbi, K.B.; El-Ashmawy, I.M.; Al-Wabel, N.A. The antidiarrheal activity of the methanol extract of some plants native to Al-Qassim Region, Saudi Arabia. J. Food Agric. Environ. 2016, 14, 239.

72. Abukhammas, A.H.; Mahyoub, J.A.; Al-Mehmadi, R.M.; Aziz, A.T.; Al-Shami, S.A. The effect of some plant extracts on mosquito Aedes aegypti (L.). Biosci. Biotechnol. Res. Asia 2014, 11, 1131-1139. [CrossRef]

73. Alqarawi, A.A.; Hashem, A.; Kumar, A.; Al-Arjani, A.B.F.; Abd_Allah, E.F.; Dar, B.A.; Wirth, S.; Davranov, K.; Egamberdieva, D. Allelopathic effects of the aqueous extract of Rhazya stricta on growth and metabolism of Salsola villosa. Plant Biosyst.-Int. J. Deal. All Asp. Plant Biol. 2018, 152, 1263-1273. [CrossRef]

74. Azab, E.; Hegazy, A.K. Monitoring the Efficiency of Rhazya stricta L. Plants in Phytoremediation of Heavy Metal-Contaminated Soil. Plants 2020, 9, 1057. [CrossRef]

75. Alvi, A.M.; Iqbal, N.; Bashir, M.A.; Rehmani, M.I.A.; Ullah, Z.; Latif, A.; Saeed, Q. Efficacy of Rhazya stricta leaf and seed extracts against Rhyzopertha dominica and Trogoderma granarium. Kuwait J. Sci. 2018, 45, 3.

76. Khan, R.A.A.; Alam, S.S.; Hayat, S.; Ahmad, M.; Ali, A.; Naz, I.; Li, Y. Eco-friendly Management of Bacterial Wilt in Tomato Using Dried Powder of the Wild Arid-Land Medicinal Shrub Rhazya stricta. Plant Dis. 2020, 104, 2613-2621. [CrossRef]

77. Anwar, T.; Ilyas, N.; Qureshi, R.; Munazir, M.; Khan, A.M.; Ansari, L.; Rahim, B.Z.; Ansari, K.A.; Panni, M.K. Allelopathic activity of solvent extracts of rhazya stricta decne. against selected weeds of wheat crop. Appl. Ecol. Environ. Res. 2018, 16, 5405-5421. [CrossRef]

78. Elhag, E.A.; Harraz, F.M.; Zaitoon, A.A.; Salama, A.K. Evaluation of some wild herb extracts for control of mosquitoes (Diptera: Culicidae). J. King Saud Univ. Agric. Sci. 1996, 8, 135-137.

79. Al-Rajhi, D.H.; Al-Hazmi, A.S.; Hussein, H.I.; Ibrahim, A.A.M.; Al-Yahya, F.A.; Mostafa, S. Nematicidal properties of Rhazya stricta and Juniperus polycarpos on Meloidogyne javanica in Saudi Arabia. Alex. Sci. Exch. 1997, 18, 135-142.

80. Iqbal, J.; Zahra, S.; Ahmad, M.; Shah, A.; Hassan, W. Herbicidal Potential of Dryland Plants on Growth and Tuber Sproutingin Purple Nutsedge (Cyperus rotundus). Planta Daninha 2018, 36. [CrossRef]

81. Al-Solami, H.M. Larvicidal activity of plant extracts by inhibition of detoxification enzymes in Culex pipiens. J. King Saud Univ. Sci. 2021, 33, 101371. [CrossRef]

82. Najoom, S.; Fozia, F.; Ahmad, I.; Wahab, A.; Ahmad, N.; Ullah, R.; Gul, A.; Bari, A.; Khan, M.Y.; Khan, A.A. Effective Antiplasmodial and Cytotoxic Activities of Synthesized Zinc Oxide Nanoparticles Using Rhazya stricta Leaf Extract. Evid. Based Complement. Altern. Med. 2021, 2021, 5586740. [CrossRef]

83. Heal, R.E. A survey of plants for insecticidal activity. Lloydia 1950, 13, 89-162. 